\title{
Numerical scheme and dynamic analysis for variable-order fractional van der Pol model of nonlinear economic cycle
}

Lei He* , Li Yi and Pei Tang

"Correspondence:

helei19850716@sina.cn

Business College, Hunan Normal

University, Changsha, China

\section{严 Springer}

\begin{abstract}
Considering the fact that the memory in economic series changes with dynamic economic environment, this paper is devoted to the proposal of a variable-order fractional van der Pol model (VOFVDPM), where the order of the derivative is replaced by a time-dependent function. A numeric scheme for this model is designed by the Adams-Bashforth-Moulton method. The dynamic behaviors of the VOFVDPM with linear and periodic variable-order functions are investigated through numerical experiment. Some dynamic characteristics of the VOFVDPM that do not exist in a fractional order van der Pol model are discovered in the numerical simulation, such as existing limit point when the linear order functions have the same ranges and opposite slopes.
\end{abstract}

MSC: $26 \mathrm{~A} 33 ; 34 \mathrm{~A} 08$

Keywords: van der Pol model; variable-order fractional derivative; dynamic behavior

\section{Introduction}

As a classical nonlinear dynamic model, the van der Pol model has attracted scholars' attention from many fields since it was proposed by the Dutch electrical engineer and physicist Balthasar van der Pol. It is extensively applied in neurology, physics, sociology, and economics. Based on Kaldor's model of business cycles, the van der Pol model of nonlinear business cycles is proposed by postulating symmetric shapes of the investment and savings functions [1]. Assuming that the investment outlay is periodic and continuous function of time, the forced van der Pol model of nonlinear economic cycles is presented in [2], which is the generalization of Goodwin's nonlinear accelerator-multiplier model. When the investment outlay is neglected, the forced van der Pol model of nonlinear economic cycles is the van der Pol model of nonlinear business cycles. In addition to Kaldor's model and Goodwin's nonlinear accelerator-multiplier model, the van der Pol model has been applied in other relevant economical problems; see [3] for more details.

The dynamic of the van der Pol model of nonlinear economic cycles is extensively studied by researchers. The bifurcation diagrams of the forced van der Pol model on varying the driver's frequency and amplitude are analyzed in [4]. It points out the coexistence of asymmetric attractors ascribed to the system symmetry of the van der Pol oscillator, and it finds that periodic, quasiperiodic, and chaotic attractors coexist. In [5], the dynamic be-

(c) $2016 \mathrm{He}$ et al. This article is distributed under the terms of the Creative Commons Attribution 4.0 International License (http://creativecommons.org/licenses/by/4.0/), which permits unrestricted use, distribution, and reproduction in any medium, provided you give appropriate credit to the original author(s) and the source, provide a link to the Creative Commons license, and indicate if changes were made. 
haviors are investigated by considering the change of control parameters, especially the pattern of bifurcation with damping parameter changing. The number of coexisting attractors in overlaps of mode-locking subzones is an important dynamic behavior, and it is analyzed in [6]. For the dynamics structure of the forced van der Pol model of nonlinear economic cycles it is found that the chaotic attractor is composed of chaotic saddles and unstable periodic orbits located in the gap regions of chaotic saddles for the model [7]. For existing chaos in the forced van der Pol model with certain parameters, the chaos control of chaotic unstable limit cycles is discussed in [8].

Inspired by the development of fractional calculus, the fractional order derivatives are applied in the forced van der Pol model of nonlinear economic cycles, for which fractional order derivatives can depict a 'memory principle' in economic dynamic. Chaos in a fractional order modified van der Pol system is studied by [9], where chaos exists in the fractional order system with the order both less than and higher than the number of the states of the integer order generalized van der Pol system. And chaos is also discovered in non-autonomous and autonomous generalized van der Pol systems excited by a sinusoidal time function with fractional orders [10]. In [11], one presents a periodically excited van der Pol system with fractional damping, and one finds that the response of the system is very sensitive to changes in the order of fractional damping. Several other papers dealing with van der Pol oscillators could be found in the literature (see [12-18] and the references therein).

Compared to the usual forced van der Pol model of nonlinear economic cycles, the contribution of the fractional forced van der Pol model of nonlinear economic cycles is that it can describe memory in economic dynamics. However, the characteristic of this memory in economic time series will change with time, for which it is affected by the macroeconomic environment and government policy at different times. Therefore, this paper will propose a variable-order fractional forced van der Pol model of nonlinear economic cycles, which can describe the fact that memory in economic series is varying with time. The orders in the fractional forced van der Pol model of nonlinear economic cycles are replaced by positive bounded continuous functions. In order to obtain an effective and applicable numerical technique for solving the variable-order fractional van der Pol model, the Adams-Bashforth-Moulton method is adopted. Then we employ the technique to obtain the numerical results for the dynamics characteristics of the variable-order fractional van der Pol model with different parameters.

The remainder of this article is organized as follows: In Section 2, we review the mathematical preliminaries as regards the variable-order fractional derivative. In Section 3, we propose a variable-order fractional forced van der Pol model of nonlinear economic cycles. In Section 4, we present the numerical technique for solving the variable-order fractional forced van der Pol model by the Adams-Bashforth-Moulton method. In Section 5, numerical experiments are performed to analyze the dynamic characteristics. The conclusions are drawn in Section 6.

\section{Variable-order fractional derivative}

In this section, we introduce some preliminaries of variable-order fractional derivative. The variable-order fractional derivative is defined by replacing the order of the fractional derivatives with a continuous bounded function in the counterparts $[19,20]$.There exist three most frequently used definitions for the general fractional differ-integral, which 
are the Grünwald-Letnikov (GL) definition, the Riemann-Liouville (RL), and the Caputo definitions. According to these definitions, their corresponding variable-order fractional derivative can be defined as follows.

Definition 1 ([19]) Let $\alpha(t)$ be a positive continuous bounded in [0,T], $f \in \mathcal{C}^{m}[0, T]$, and $m=\left[\max _{0 \leq t \leq T} \alpha(t)\right]+1$. Then

$$
D_{G L}^{\alpha(t)} f(t)=\lim _{h \rightarrow 0} \frac{1}{h^{\alpha(t)}} \sum_{i=0}^{N}(-1)^{i}\left(\begin{array}{c}
\alpha(t) \\
i
\end{array}\right) f(t-i h),
$$

with $h=t / N$ being called the GL variable-order fractional derivative of $f(t)$.

Definition $2([19,20])$ The Riemann-Liouville variable-order fractional derivative of $f(t)$ for a continuously differentiable function $f:[a,+\infty) \rightarrow \mathbb{R}$ is defined as

$$
D_{R L}^{\alpha(t)} f(t)=\frac{1}{\Gamma(m-\alpha(t))} \frac{d^{m}}{d t^{m}} \int_{a}^{t}(t-s)^{m-\alpha(s)-1} f(s) d s,
$$

where $m-1 \leq \alpha(t)<m m=\left[\max _{0 \leq t \leq T} \alpha(t)\right]+1$.

Definition $3([19,20])$ The Caputo variable-order fractional derivative of $f(t)$ for a continuously differentiable function $f:[0,+\infty) \rightarrow \mathbb{R}$ is defined as

$$
D_{C}^{\alpha(t)} f(t)=\frac{1}{\Gamma(m-\alpha(t))} \int_{a}^{t}(t-s)^{m-\alpha(t)-1} f^{(m)}(s) d s,
$$

where $m-1 \leq \alpha(t)<m, m=\left[\max _{0 \leq t \leq T} \alpha(t)\right]+1$.

Remark 1 For the above definitions of variable-order fractional derivative, when $\alpha(t)=$ $q>0$, (1), (2), (3) describe the fractional derivative. If $\alpha(t)=m-1,(1),(2)$, (3) describe the classical $(m-1)$ th order derivative. If $\alpha(t)=0, D_{G L, R L, C}^{\alpha(t)} f(t)=f(t)$.

The main advantage of the Caputo derivative is that the initial conditions for the fractional differential equations are of the same form as that of the integer order differential equations [21]. Therefore, we study the VOFVDPM with the Caputo derivative in this paper.

\section{Variable-order fractional van der Pol model of nonlinear business cycles}

The forced van der Pol model of nonlinear business cycles can be described as follows:

$$
\frac{d^{2} x}{d t^{2}}+\mu\left(x^{2}-1\right) \frac{d x}{d t}+x=a \sin (\omega t)
$$

where $x$ is economic variable, such as national income; $\mu(>0)$ is the coefficient on nonlinear damping term and determines the speed of adjustment; $a$ is the amplitude of the exogenous force, and $\omega$ is the frequency of the exogenous force; $a \sin (\omega t)$ is called the force function, and it can be explained as the amount of autonomous investment outlays at $t$ [2]. When $a=0$, (4) is the unforced van der Pol model of nonlinear business cycles. 
Based on (4), we present the variable-order forced van der Pol model (VOFVDPM) of nonlinear business cycles as follows:

$$
{ }_{0} D_{t}^{\alpha(t)}{ }_{0} D_{t}^{\beta(t)} x+\mu\left(x^{2}-1\right)_{0} D_{t}^{\beta(t)} x+x=a \sin (\omega t)
$$

where $\alpha(t), \beta(t)$ are positive continuous bounded functions at $[0, T]$.

Remark 2 If $\alpha(t)=\alpha, \beta(t)=\beta$, (5) is a forced variable-order fractional van der Pol model of nonlinear business cycles. If $\alpha(t)=1$, and $\beta(t)=1$, the system (5) is a forced van der Pol model of nonlinear business cycles.

Remark 3 If $a=0$, (5) is an unforced variable-order fractional van der Pol model of nonlinear business cycles.

Let $y=D_{t}^{\beta(t)} x, q_{1}(t)=\beta(t), q_{2}(t)=\alpha(t)$ then (5) can be rewritten as a two dimensional variable-order fractional system,

$$
\begin{aligned}
& { }_{0} D_{t}^{q_{1}(t)} x=y, \\
& { }_{0} D_{t}^{q_{2}(t)} y=\mu\left(1-x^{2}\right) y-x+a \sin (\omega t) .
\end{aligned}
$$

For the VOFVDPM, $x$ is the macroeconomic variable of a certain economy, such as national income, GDP, and so on. $a \sin (\omega t)$ is for the periodical investment outlays, which is a controllable variable and has impacts on the macroeconomic variable $x$. The variableorder fractional derivative of VOFVDPM reflects the varying memory in the macroeconomic series $x$. So the order functions $q_{1}(t), q_{2}(t)$ depict the evolution path of the memory in the macroeconomic series $x$. Therefore, VOFVDPM with a certain variable-order fractional derivative not only depicts the complex dynamics of national income, but also reflects the varying memory in the economical series of the national income. The application of a variable-order fractional derivative in the van der Pol model can be extended to other dynamic economic models.

\section{Numerical scheme}

The Adams-Bashforth-Moulton method is a type of predictor-corrector methods and a relatively new approach to provide a numerical approximation to solve the fractional order differential equations. It is studied and discussed thoroughly in [22, 23]. In this paper, we adapt the Adams-Bashforth-Moulton method to solve the variable-order fractional van der Pol model (VOFVDPM).

Consider (6) with $0<q_{i}(t) \leq 1, t \geq 0, i=1,2$, and the initial condition $(x(0), y(0))=$ $\left(x_{0}, y_{0}\right)$. We select the following uniform grid:

$$
h=\frac{T}{N}, \quad t_{n}=n h,
$$

where $n=0,1,2, \ldots, N$, and $t_{0}=0, t_{N}=T$. Then model (6) can be discretized as follows:

$$
\left\{\begin{aligned}
x_{n+1}= & x_{0}+\frac{h^{q_{1}\left(t_{n+1}\right)}}{\Gamma\left(q_{1}\left(t_{n+1}\right)+2\right)} x_{n+1}^{p}+\sum_{j=0}^{n} \frac{h^{q_{1}\left(t_{n+1}\right)} \gamma_{1, j, n+1}}{\Gamma\left(q_{1}\left(t_{n+1}\right)+2\right)} x_{j} \\
y_{n+1}= & y_{0}+\frac{h^{2}\left(t_{n+1}\right)}{\Gamma\left(q_{2}\left(t_{n+1}\right)+2\right)}\left(\mu\left(x_{n+1}^{p}-1\right) y_{n+1}^{p}-x_{n+1}^{p}+a \sin \left(\omega\left(t_{n}+1\right)\right)\right) \\
& +\sum_{j=0}^{n} \frac{h^{2}\left(t_{n+1}\right)}{\Gamma\left(q_{2}\left(t_{n+1}\right)+2\right)}\left(\mu\left(x_{j}^{2}-1\right) y_{j}-x_{j}+a \sin \left(\omega\left(t_{j}\right)\right)\right)
\end{aligned}\right.
$$


where

$$
\left\{\begin{array}{l}
x_{n+1}^{p}=x_{0}+\sum_{j=0}^{n} \frac{\theta_{1, j, n+1}}{\Gamma\left(q_{1}\left(t_{n+1}\right)\right)} x_{j}, \\
y_{n+1}^{p}=y_{0}+\sum_{j=0}^{n} \frac{\theta_{2, j, n+1}}{\Gamma\left(q_{2}\left(t_{n+1}\right)\right)}\left(\mu\left(x_{j}^{2}-1\right) y_{j}-x_{j}+a \sin \left(\omega\left(t_{j}\right)\right)\right),
\end{array}\right.
$$

and the coefficients are calculated by

$$
\gamma_{i, j, n+1}= \begin{cases}n^{q_{1}\left(t_{n}+1\right)+1}-\left(n-q_{i}\left(t_{n+1}\right)\right)(n+1)^{q_{i}\left(t_{n+1}\right)}, & j=0, \\ (n-j-2)^{q_{i}\left(t_{n+1}\right)+1}+(n-j)^{q_{i}\left(t_{n+1}\right)+1} & \\ -2(n-j+1)^{q_{i}\left(t_{n+1}\right)+1}, & 1 \leq j \leq n, \\ 1, & j=n+1,\end{cases}
$$

and

$$
\theta_{i, j, n+1}=\frac{h^{q_{i}\left(t_{n+1}\right)}}{q_{i}\left(t_{n+1}\right)}\left((n-j+1)^{q_{i}\left(t_{n+1}\right)}-(n-j)^{q_{i}\left(t_{n+1}\right)}\right),
$$

and $i=1,2$ for (10) and (11).

\section{Numerical results}

In this section, we will analyze the dynamic behaviors of VOFVDPM by the above numerical scheme. The cases where the orders of the derivatives in VOFVDPM are varying with different linear and periodical functions of time are investigated. The experiments under $a=0$ are performed. Figures 1-11 are numerical results of VOFVDPM (6) for the cases where the variable-order functions $q_{1}, q_{2}$ are linear and periodic. We select the following uniform grid: $T=300(t \in[0,300]), h=0.02, N=15,000, t_{n}=0.02 n, t_{0}=0, t_{N}=300$.

Figures 1(a), (b) exhibit the phase plots for linear variable-order functions $q_{1}(t)=q_{2}(t)$, which are linear decreasing and increasing in the same range $[0.9,1]$ with the same speed, $1 / 3,000$, respectively. It shows that the responses of the VOFVDPM with linear decreasing order function approach a limit cycle faster than with a linear increasing order function. The shapes of the limit cycles with linear decreasing and increasing variable-order functions are different in spite of the same range and speed. Figure 2 shows the phase plots

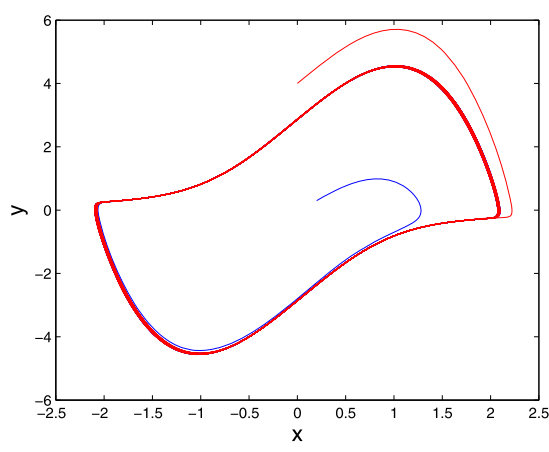

(a) $q_{1}(t)=q_{2}(t)=1-1 t / 3,000$

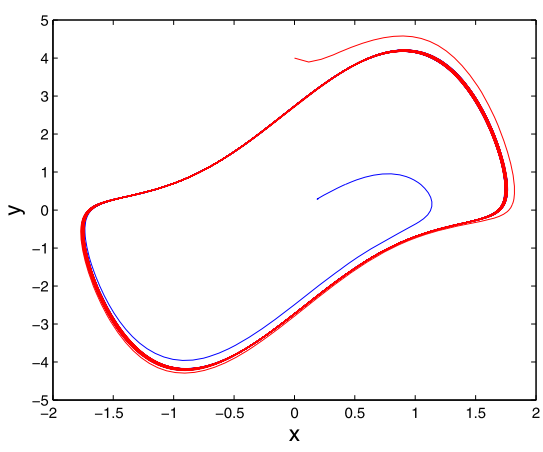

(b) $q_{1}(t)=q_{2}(t)=0.9+t / 3,000$

Figure 1 Phase plots for the VOFVDPM with $q_{1}(t)=q_{2}(t)=1-1 t / 3,000$ and $q_{1}(t)=q_{2}(t)=0.9+1 t /$ 3,000 (the other common parameters are $a=0, \mu=2.5$ ) [blue line: $(x(0), y(0))=(0.2,0.3)$; red line: $(x(0), y(0))=(0,4)]$. 
Figure 2 Phase plot for the VOFVDPM with different linear variable-order functions, $a=0, \mu$ $=2.5$, and $(x(0), y(0))=(1.3,1.2)$.

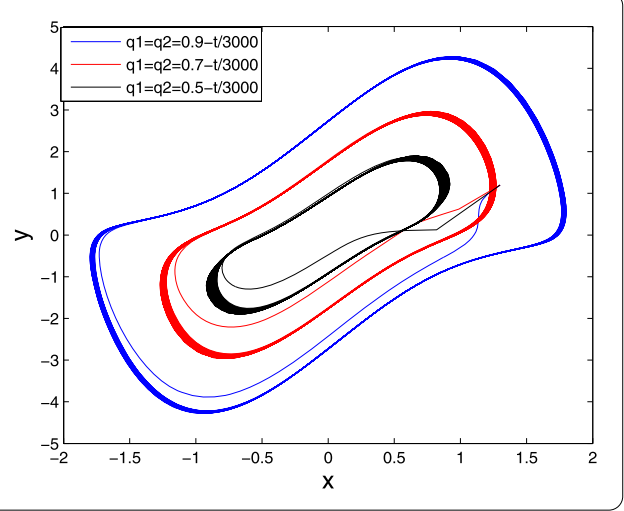

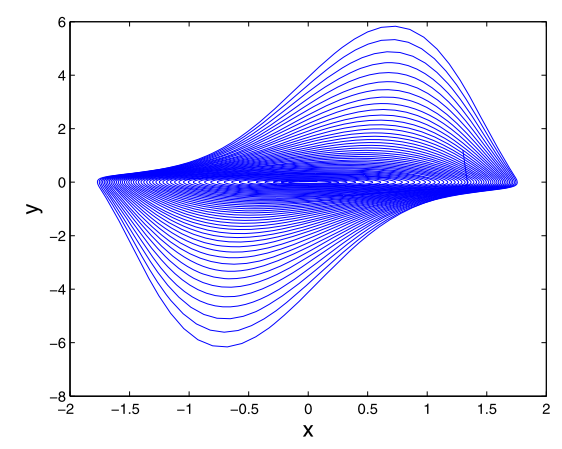

(a) $y(t)$ vs. $x(t)$

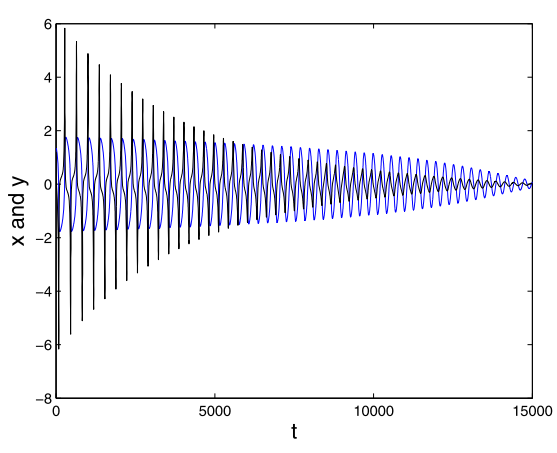

(b) trajectories of $x$ and $y$

Figure 3 Phase plot and trajectories (blue line: $x(t)$, black line: $y(t)$ ) for the VOFVDPM with $q_{1}(t)=1-4 t / 3,000, q_{2}(t)=0.6+4 t / 3,000, a=0, \mu=2.5$, and $(x(0), y(0))=(1.3,1.2)$.

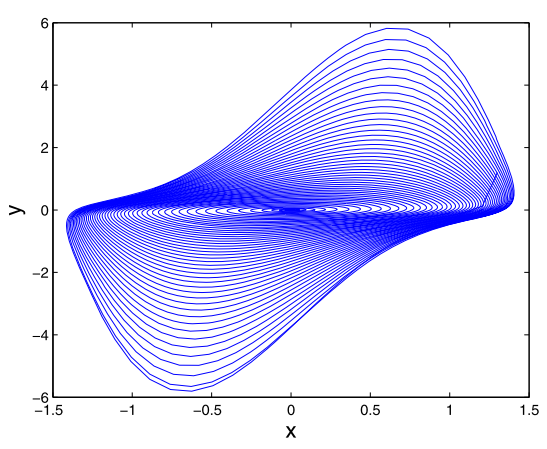

(a) $y(t)$ vs. $x(t)$

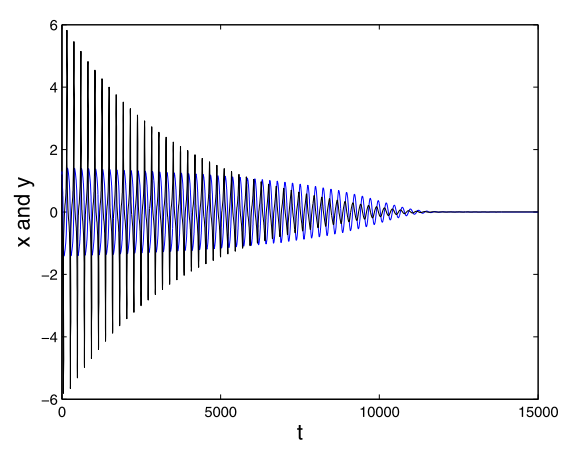

(b) trajectories of $x$ and $y$

Figure 4 Phase plot and trajectories (blue line: $x(t)$, black line: $y(t))$ for the VOFVDPM with $q_{1}(t)=0.9-4 t / 3,000, q_{2}(t)=0.5+4 t / 3,000, a=0, \mu=2.5$, and $(x(0), y(0))=(1.3,1.2)$.

for linear variable-order functions with different intercepts. From it, we can find that the scale of the limit cycles becomes smaller with smaller intercepts in the linear variableorder functions.

In order to investigate the dynamic characteristics of the VOFVDPM with $q_{1}$ not being equal to $q_{2}$, Figures 3-5 exhibit the phase plots and trajectories for the case: $q_{1}(t)=$ $1-4 t / 3,000, q_{2}(t)=0.6+4 t / 3,000$, the case: $q_{1}(t)=0.9-4 t / 3,000, q_{2}(t)=0.5+4 t / 3,000$, 


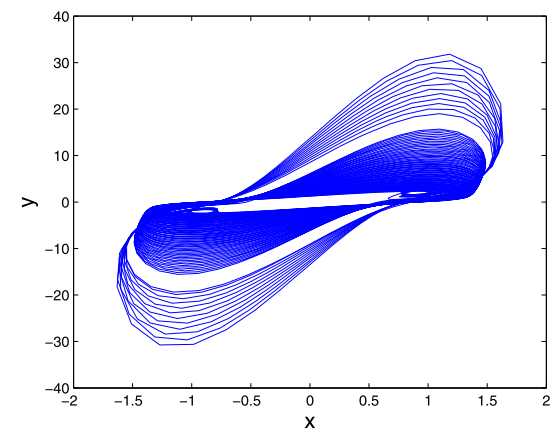

(a) $y(t)$ vs. $x(t)$

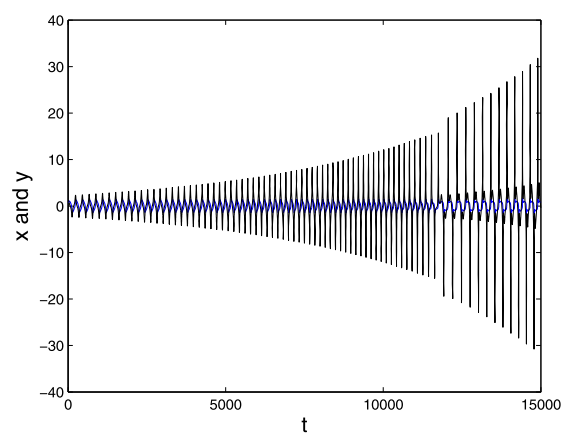

(b) trajectories of $x$ and $y$

Figure 5 Phase plot and trajectories (blue line: $x(t)$, black line: $y(t)$ ) for the VOFVDPM with $q_{1}(t)=1-4 t / 3,000, q_{2}(t)=0.6+4 t / 3,000, a=0, \mu=2.5$, and $(x(0), y(0))=(1.3,1.2)$.

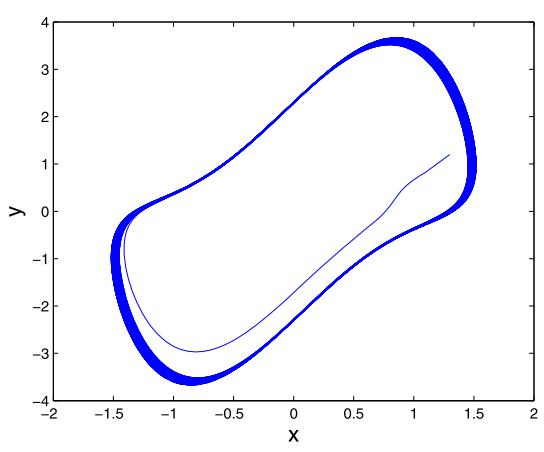

(a) $q_{1}=q_{2}=0.8+0.1 \sin (t / 25)$

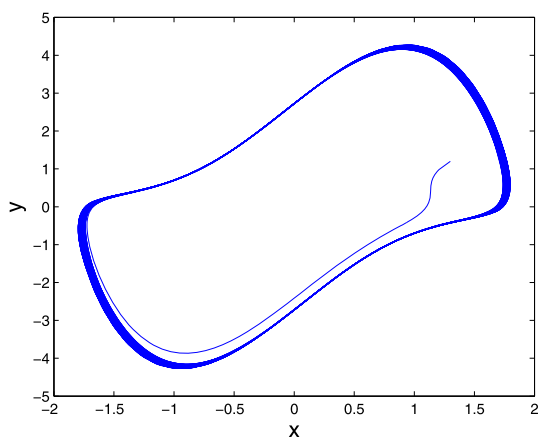

(c) $q_{1}=q_{2}=0.9+0.1 \sin (t / 25)$

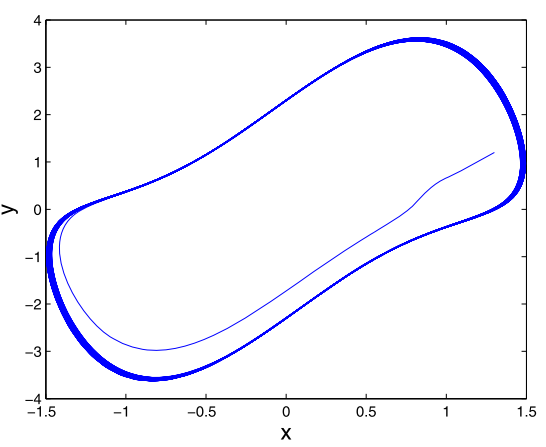

(b) $q_{1}=q_{2}=0.8+0.05 \sin (t / 25)$

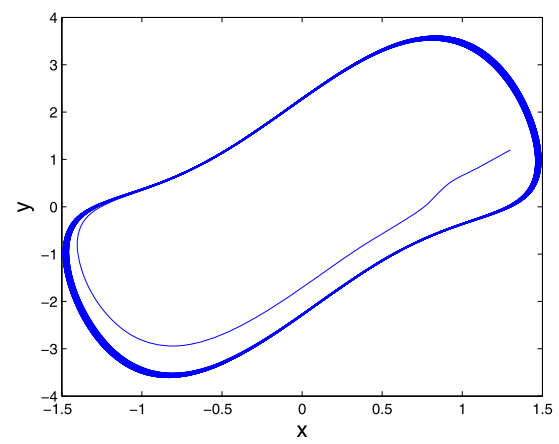

(d) $q_{1}=q_{2}=0.8+0.05 \sin (t / 5)$

Figure 6 Phase plot for the VOFVDPM with different periodical functions, $a=0, \mu=2.5$, and $(x(0), y(0))=(1.3,1.2)$.

and the case: $q_{1}(t)=0.6+4 t / 3,000, q_{2}(t)=1-4 t / 3,000$. For Figure $3, q_{1}, q_{2}$ have the same range $[0.6,1]$, but decrease and increase with opposite slopes, respectively. It shows that the responses of the VOFVDPM have convergence to the limit point $(0,0)$. Figure 4 for linear $q_{1}, q_{2}$ with the same ranges $[0.5,0.9]$ and opposite slopes, also has the same dynamic behaviors and faster reaches the limit point $(0,0)$ than the case in Figure 3 . However, the trajectories are divergent when $q_{1}, q_{2}$ increase and decrease in the same range with opposite slopes, respectively (Figure 5). 


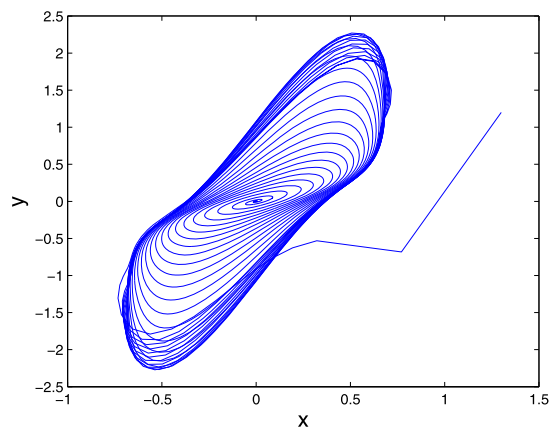

(a) $y(t)$ vs. $x(t)$

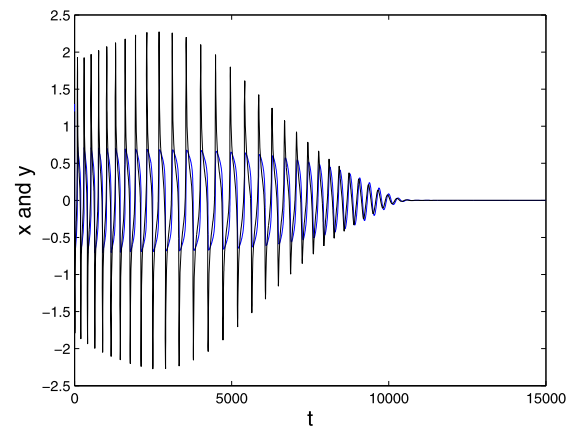

(b) trajectories of $x$ and $y$

Figure 7 Phase plot and trajectories (blue line: $x(t)$, black line: $y(t))$ for the VOFVDPM with $q_{1}(t)=0.4+0.1 \sin (t / 50), q_{2}(t)=0.3+2 t / 3,000, a=0, \mu=2.5$, and $(x(0), y(0))=(1.3,1.2)$.

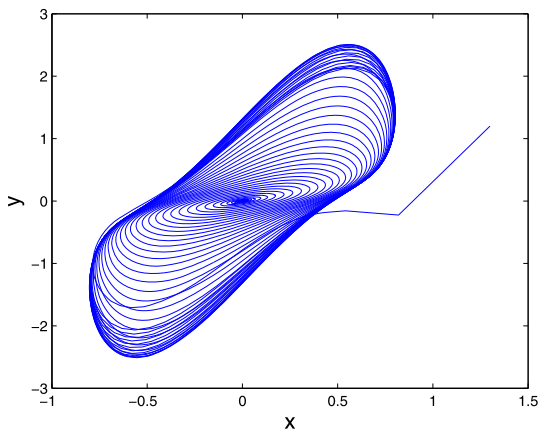

(a) $y(t)$ vs. $x(t)$

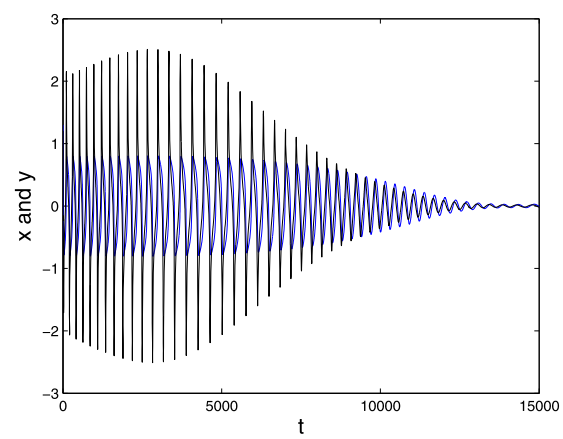

(b) trajectories of $x$ and $y$

Figure 8 Phase plot and trajectories (blue line: $x(t)$, black line: $y(t))$ for the VOFVDPM with $q_{1}(t)=0.5+0.1 \sin (t / 50), q_{2}(t)=0.4+2 t / 3,000, a=0, \mu=2.5$, and $(x(0), y(0))=(1.3,1.2)$.

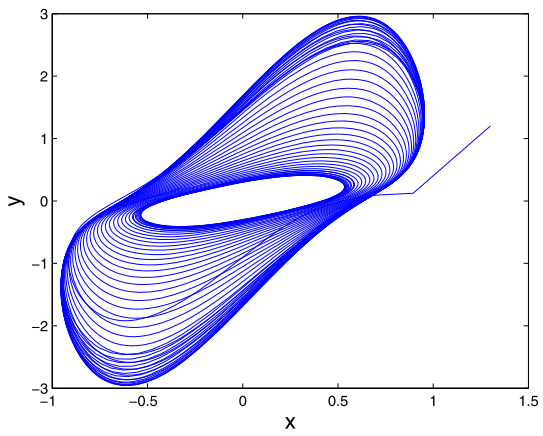

(a) $y(t)$ vs. $x(t)$

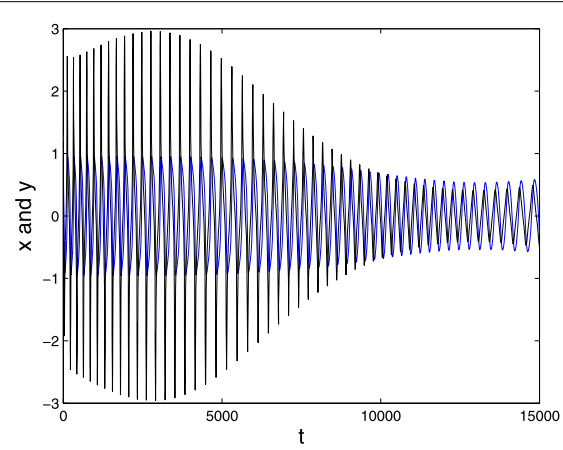

(b) trajectories of $x$ and $y$

Figure 9 Phase plot and trajectories (blue line: $x(t)$, black line: $y(t))$ for the VOFVDPM with $q_{1}(t)=0.6+0.1 \sin (t / 50), q_{2}(t)=0.5+2 t / 3,000, a=0, \mu=2.5$, and $(x(0), y(0))=(1.3,1.2)$.

Figure 6 shows the phase plots for the VOFVDPM with the orders of derivative being different periodic order functions. From it, we find that the responses of them reach a limit cycle. Comparing Figures 6(a) to (b), the scale of the limit cycle with wider amplitude of the order functions are larger. Figure $6(\mathrm{~b})$ and (d) have a periodic variable-order 


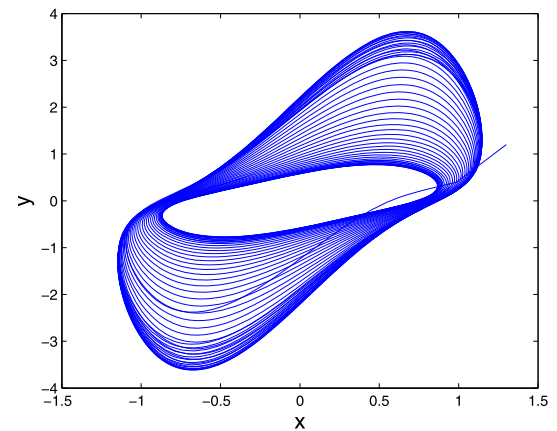

(a) $y(t)$ vs. $x(t)$

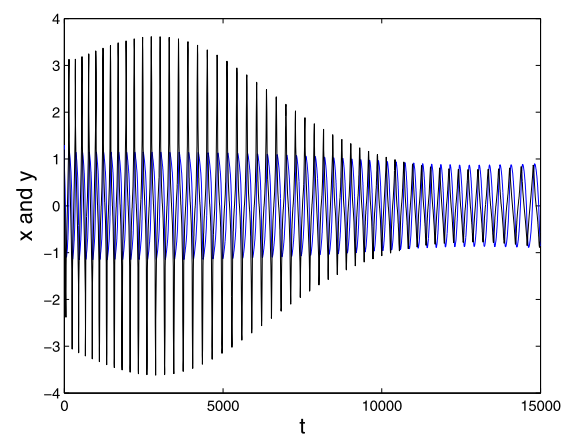

(b) trajectories of $x$ and $y$

Figure 10 Phase plot and trajectories (blue line: $x(t)$, black line: $y(t))$ for the VOFVDPM with $q_{1}(t)=0.7+0.1 \sin (t / 50), q_{2}(t)=0.6+2 t / 3,000, a=0, \mu=2.5$, and $(x(0), y(0))=(1.3,1.2)$.

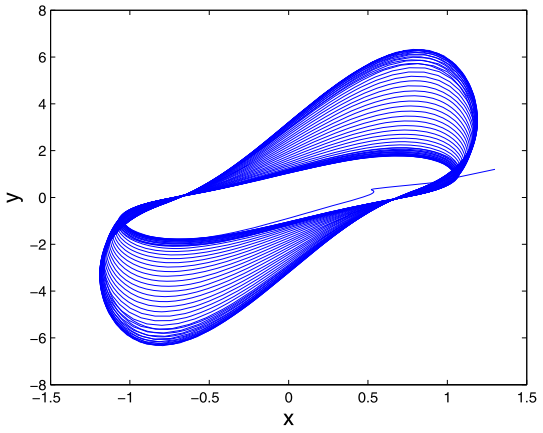

(a) $y(t)$ vs. $x(t)$

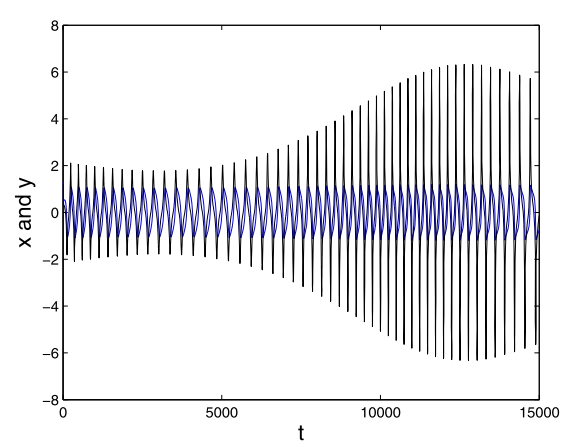

(b) trajectories of $x$ and $y$

Figure 11 Phase plot and trajectories (blue line: $x(t)$, black line: $y(t))$ for the VOFVDPM with $q_{1}(t)=0.6+2 t / 3,000, q_{2}(t)=0.7+0.1 \sin (t / 50), a=0, \mu=2.5$, and $(x(0), y(0))=(1.3,1.2)$.

function with different periods, but it seems that significant differences are not discovered yet. Figures 6(a) and (c) exhibit different shapes of the limit cycle when the ranges of periodical variable-order functions are different. From Figure 6, the periodic variable-order functions have little effect on the responses of the VOFVDPM.

Figures 7-11 present the phase plots and trajectories of the VOFVDPM with $q_{1}, q_{2}$ being linear and periodic functions. The order functions $q_{1}, q_{2}$ are periodic and linear, respectively, in the same range for Figures $7-10$, where the ranges are $[0.3,0.5],[0.4,0.6]$, $[0.5,0.7],[0.6,0.8]$. We can find that the responses of the model approach the point $(0,0)$ in Figures 7-8 with smaller orders, and they are in convergence to a limit cycle in Figures 9-10 with larger orders. However, Figure 11 shows that the trajectories are divergent, where the order functions $q_{1}, q_{2}$ are linear and periodic, respectively, in the same range, $[0.6,0.8]$.

The above numerical experiments investigate the dynamic behaviors of the VOFVDPM with different linear and periodical functions in the case $a=0$. It shows that some novel dynamic characteristics of the model have been found in the numerical results, such as the existence of a limit point $(0,0)$ when linear $q_{1}, q_{2}$ are in the same ranges and have opposite slopes. These novel dynamic characteristics indicate that the limit cycle is not the only 
characteristic of the FVDP model when the order of the derivative is a time-dependent function.

\section{Conclusion}

In this paper, we successfully extend the forced van der Pol model with nonlinear economic cycle to the VOFVDPM, which considers the memory in an economic series varying with time. The numeric scheme based on the Adams-Bashforth-Moulton method for the VOFVDPM has been designed. By the scheme, the dynamic behaviors of the model with linear or periodic variable-order function have been analyzed. The results of the analysis suggest that the VOFVDPM presents some novel dynamic characteristics, which cannot be found in an integral and constant fractional van der Pol model.

\section{Competing interests}

The authors declare that there is no conflict of interests, nor are there financial or competing interests regarding the publication of this article.

\section{Authors' contributions}

All authors contributed equally to the manuscript and typed, read, and approved the final manuscript

\section{Acknowledgements}

The project supported by National Natural Science Foundation of China (71501070) and Education Department foundation of Hunan Province (15B150).

Received: 6 April 2016 Accepted: 11 July 2016 Published online: 25 July 2016

\section{References}

1. Chang, WW, Smyth, DJ: The existence and persistence of cycles in a non-linear model: Kaldor's model re-examined. Rev. Econ. Stud. 38(1), 37-44 (1971)

2. Lorenz, HW: Goodwin's nonlinear accelerator and chaotic motion. J. Econ. 47, 413-418 (1987)

3. Chian, ACL: Nonlinear dynamics and chaos in macroeconomics. Int. J. Theor. Appl. Finance 3, 601-624 (2000)

4. Parlitz, U, Lauterborn, W: Period-doubling cascades and devil's staircases of the driven van der Pol oscillator. Phys. Rev. A 6, 1428-1434 (1987)

5. Mettin, R, Parlitz, U, Lauterborn, W: Bifurcation structure of the driven van der Pol oscillator. Int. J. Bifurc. Chaos 3, 1529-1555 (1993)

6. Xu, JX, Jiang, J: The global bifurcation characteristics of the forced van der Pol oscillator. Chaos Solitons Fractals 3(7), 3-19 (1996)

7. Chian, ACL, Rempel, EL, Rogers, C: Complex economic dynamics: chaotic saddle, crisis and intermittency. Chaos Solitons Fractals 29, 1194-1218 (2006)

8. Mahmound, GM, Farghaly, AAM: Chaos control of chaotic limit cycles of real and complex van der Pol oscillators. Chaos Solitons Fractals 21, 915-924 (2004)

9. Ge, ZM, Zhang, AR: Chaos in a modified van der Pol system and in its fractional order systems. Chaos Solitons Fractals $32,1791-1822(2006)$

10. Ge, ZM, Hsu, MY: Chaos in a generalized van der Pol system and in its fractional order systems. Chaos Solitons Fractals $33,592-604(2007)$

11. Chen, $\mathrm{JH}$, Chen, WC: Chaotic dynamics of the fractionally damped van der Pol equation. Chaos Solitons Fractals 35 , 188-198 (2008)

12. Barbosa, RS, Machado, JAT, Vingare, BM, Calderon, AJ: Analysis of the van der Pol oscillator containing derivatives of fractional order. J. Vib. Control 13, 1291-1301 (2007)

13. Ge, ZM, Hsu, MY: Chaos excited chaos synchronizations of integral and fractional order generalized van der Pol systems. Chaos Solitons Fractals 36, 592-604 (2008)

14. Tavazoei, MS, Haeri, M, Attari, M, Siami, M, Bolouki, S: More details on analysis of fractional order van der Pol oscillator. J. Vib. Control 15, 803-819 (2009)

15. Attari, M, Mohammad, MH, Tavazoei, S: Analysis of a fractional order van der Pol-like oscillator via describing function method. Nonlinear Dyn. 61, 265-274 (2010)

16. Suchorsky, MK, Rand, RH: A pair of van der Pol oscillators coupled by fractional derivatives. Nonlinear Dyn. 69 313-324 (2012)

17. $\mathrm{Xu}, \mathrm{Y}$, Agrawal, OP: Models and numerical schemes for generalized van der Pol equations. Commun. Nonlinear Sci. Numer. Simul. 18, 3575-3589 (2013)

18. Mishra, V, Das, S, Jafari, H, Ong, SH: Study of fractional order van der Pol equation. J. King Saud Univ., Sci. 28, 55-60 (2016)

19. $\mathrm{Ma}, \mathrm{S}, \mathrm{Xu}, \mathrm{Y}, \mathrm{Yue}, \mathrm{W}$ : Numerical solution of a variable-order fractional financial system. J. Appl. Math. 2012, Article ID 417942 (2012). doi:10.1155/2012/417942(2012)

20. Valerio, D, Costa, JS: Variable-order fractional derivatives and their numerical approximations. Signal Process. 3(91), 470-483 (2011) 
21. $\mathrm{Xu}, \mathrm{Y}, \mathrm{He}, \mathrm{Z}$ : Synchronization of variable-order fractional financial system via active control and method. Cent. Eur. J. Phys. (2013). doi:10.2478/s11534-013-0237-x

22. Diethelm, K: The Analysis of Fractional Differential Equations. Springer, Berlin (2010)

23. Diethelm, K, Ford, NJ, Freed, AD: A predictor-corrector approach for the numerical solution of fractional differential equations. Nonlinear Dyn. 29, 3-22 (2002)

Submit your manuscript to a SpringerOpen ${ }^{\odot}$ journal and benefit from:

- Convenient online submission

Rigorous peer review

- Immediate publication on acceptance

- Open access: articles freely available online

- High visibility within the field

- Retaining the copyright to your article

Submit your next manuscript at $\boldsymbol{s p r i n g e r o p e n . c o m ~}$ 